\title{
Satisfaction on Sports Learning Altitude Based on Structure Equation Model in Secondary School
}

\author{
Hongjiang Wang \\ Chengdu Sport University, Chengdu, 610041, China
}

Keywords: Structure Equation Model; satisfaction of learning; learning attitude; association.

\begin{abstract}
The purpose of this study was to investigate the association relationship between learning satisfaction and attitude at middle school in Southwest China. Students in 15 secondary schools in Sichuan and Chongqing have been surveyed, the exploratory and confirmatory factors have been used to establish structure equation model. The results indicated that 1) the overall learning satisfaction at physical education class in middle school in Southwest China embodied in five factors: teaching ability, facilities and equipment, safety for teaching, sports policy, and classroom atmosphere. In terms of scoring from separate dimensions, the classroom atmosphere scored the highest score, while the school's sports policy scored the lowest score; there is no difference on sport policy in scores on the school location in five factors, 2) the overall learning attitude toward physical education class at secondary school in southwest China embodied in three areas, according to the size of the contribution rate: movement awareness, sport emotion, and exercise behavior; and 3) there were significant positive correlation between teaching ability, facilities and equipment, safety for teaching, sports policy, and classroom atmosphere and movement awareness, sport emotion and exercise behavior. The findings of this study revealed that there was a significant positive effect of learning satisfaction on learning attitude at secondary school students in southwest China, the standardized regression coefficient of regression was 0.58 .
\end{abstract}

\section{Introduction}

In the last two decades, there are few research literature on learning satisfaction of adolescents in PE classes. In this study, with reference to study recommendations of relevant scholars, two measurement scales are established, namely the scales of learning satisfaction and learning attitude in PE classes; in addition, Exploratory Factor Analysis (EFA) and Confirmatory Factor Analysis (CFA) are combined to explore the potential structures between variables, so as to establish assumptions and verify the presence or not of certain connection between learning satisfaction and learning attitude in PE classes. It is desired that the study results can provide some useful help to theories and teaching practices to physical teachers.

\section{Subjects and methods}

\subsection{Respondent}

The high school students in Nanchong, Sichan and Yongchuan, Chongqing were selected as respondents. Based on the school distribution data provided by Education Bureau of the two cities and sorted by the distance from the school to the downtown, schools were separated into two categories: schools in downtown and schools in suburbs. 15 schools in total were selected by the balanced random sampling, including 7 in Nanchong (4 downtowns and 3 suburbs) and 8 in Chongqing ( 4 downtowns and suburbs respectively). Taking class as a unit, 4 classes (senior grade one and grade two only, senior grade three excluded) were randomly selected from each sampled school, 60 classes selected in total.

\subsection{Questionnaire Design}

The questionnaire includes the basic personal information of students (such as gender, grade, school characteristics and family background) and two scales, where the setting of scales contained some references from study and design of related scholars. 
In the questionnaire of learning satisfaction of high school students in PE classes, 25 entries are set up in total, and the scoring adopted is the likert-type 5-point scoring. Based on the students' learning satisfaction situation, there are five multiple choice answers in one entry: "very satisfied", "satisfied", "normal", "dissatisfied" and "very dissatisfied", and the score of each choice is 5, 4, 3, 2 and 1 point in order. The higher the score, the higher the learning satisfaction in PE classes is, and vice versa.

In the questionnaire of learning altitude of high school students in PE classes, 18 entries are set up in total, and the scoring adopted is also the likert-type five-point scoring. The subject answers questions as per his or her actual experience and the degree changes from "disagree completely", "disagree", "partly agree", "agree" and "agree completely", with the respective points of 1, 2, 3, 4 and 5.

\subsection{Questionnaire Distribution and Collection}

Members of the Research Group have got in touch with the physical teachers of corresponding schools and directly arranged PE teachers of related classes to be responsible for questionnaire distribution and collection. Total 2511 copies of questionnaires were distributed, 1115 in Nanchong (1115 collected) and 1396 in Chongqing (1384 collected).

\subsection{Mathematical Statistics}

The SPSS16.0 and Amos17.0 Version are used for statistical analysis; EFA and CFA factors are combined; Cronbach's $\alpha$ reliability analysis and ANOVA are conducted; for all statistics, the significant level was set at $\alpha=0.05$.

\section{Result and Analysis}

\subsection{Construction of Learning Satisfaction Dimensions in PE Classes}

The extracted data on common factors of learning satisfaction of high school students in PE classes showed that the KMO value was 0.89 , Bartlett spherical test chi-square value $\mathrm{x}^{2}$ was 806.24 and $\mathrm{P}$ was less than 0.05 . This indicated the spherical hypothesis was rejected, indicating the entries setting in the satisfaction measurement scale were suitable for factor analysis.

Taking the characteristic value 1 as the sampling standard, five common factors are extracted from the learning satisfaction scale of high school students in PE classes, with a cumulative contribution rate of $68.58 \%$. The first common factor contains three entries and has the highest contribution rate $(23.24 \%)$, mainly related to the demonstration ability, professional teaching skills and motivation of PE teachers. Therefore, the first factor is named as "teaching ability" factor. The second common factor contains four entries, with a contribution rate of $18.57 \%$, mainly dealing with the quality, quantity and space of grounds and facilities used in PE classes, hence named as "grounds and equipment" factor. The third common factor covers three entries, with a contribution rate $13.95 \%$, mainly related to safety and injury treatment aspects of PE classes, hence named as "teaching safety" factor. The fourth one includes five entries, with a contribution rate of $9.71 \%$, mainly related PE curriculum arrangement, importance to school, relevant specifications and equipment rental and other relevant systems, hence named as "sports policy" factor. The fifth common factor have three entries, with a contribution rate of $5.59 \%$, mainly related to mutual assistance, competition and care between students in PE classes, hence named as "classroom atmosphere" factor. There are 25 entries in the original questionnaire. In this study, five common factors are extracted; after using the orthogonal rotation to obtain the variable loads, seven entries with relatively small contributions are omitted and 18 entries are left ultimately.

The maximum likelihood method was adopted to verify and analyze the raw data matrix of the satisfaction measurement model. The confirmatory factory analysis showed that in the study scale (including 18 entries), EA was equal to .08 . Values of these indicators have reached acceptable standards, and thus it was reasonable to consider the satisfaction measurement model having good construct validity. In addition, the reliability indexes of all five dimensions are in the range of $0.71-0.86$, all greater than the standard value 0.6 , while the overall composite reliability is 0.93 , also 
meeting the convergent validity requirement. Therefore, it is considered that the satisfaction measurement scale has a good quality.

\subsection{Construction of Learning Attitude Dimensions in PE Classes}

The extraction and analysis of common factors of learning attitude of high school students in PE classes show: KMO is equal to 0.91, Bartlett spherical test chi-square value $\mathrm{x} 2$ is 789.45 and the corresponding probability $\mathrm{P}$ is less than 0.001 for all factors. The spherical hypothesis is rejected, indicating the scale is suitable for factor analysis. Likewise, three common factors are extracted with the characteristic value I as the sampling standard, with a cumulative contribution rate of $72.43 \%$. The first common factor has five entries, with the highest contribution rate (35.34\%), mainly related to the value awareness of PE classes, such as the development of athletic potential, establishment of fitness concepts, improvement of physical abilities and skills and prevention of sports injury, hence named "sports awareness" factor. The second common factor contains five entries, with a contribution rate of $20.54 \%$, mainly related to stress relief by PE classes, learning cooperation with classmates, enhancement of students' feelings and meeting demands of self-interests, thus named as "sports emotion" factor. The third common factor covers five entries, with a contribution rate of $16.55 \%$, mainly related to students achieving self-athletic performance via PE classes, such as demonstrations, Q \& A and initiative exercises, hence named as "sports behavior" factor. There are 18 entries in the original questionnaire. Three entries are omitted due to too low contribution rates in the extracted common factors, with 15 entries left only.

The CFA results of the scale (including 15 entries) showed: $E A=007$. Values of these indicators have reached an acceptable standard, and thus it is reasonable to consider the learning altitude measurement model having good construct validity. In addition, the reliability indexes of all three dimensions are in the range of 0.76-0.87, all greater than the standard value 0.6 , while the overall composite reliability is 0.89 , also meeting the convergent validity requirement. Therefore, it is considered that the altitude measurement scale has a good quality.

\subsection{Construction of Structure Model for Learning Satisfaction and Attitude in PE Classes}

The $\mathrm{X}^{2} / \mathrm{DF}$ of the model is equal to 1.48 , and $\mathrm{P}$ is equal to 0.19 , greater than 0.05 . Prompt: suppose the model fits the original data matrix well. In addition, values of CFI, GFI, NFI, NNFI, IFI, and RMSEA are $0.912,0.924,0.917,0.935,0.927$ and 0.094 in order, all up to an acceptable standard. In short, the fitting goodness of the structure model is satisfactory, showing the hypothesized model fits the observed data well in this survey.

\section{Analyses and Discussions}

The PE learning satisfaction of high school students in Southwest China can be divided into five dimensions. Seeing from the contribution rates of extracted factors, the five dimensions are "teaching ability" of PE teachers (23.24\%), "grounds and equipment" (18.57\%), "teaching safety" (13.95\%), "sports policy" (9.71\%) and "classroom atmosphere" (5.59\%) in order. Data analysis indicated that the scores of 5 dimensions are $3.14 \pm 1.21,3.28 \pm 1.16,2.97 \pm 1.17,2.94 \pm 1.23$ and $3.32 \pm 1.04$ respectively, basically above the average. Obviously, "classroom atmosphere" scored the highest, while the "sports policy" of school the lowest. Furthermore, except the "sports policy" of which the score has no discrepancy caused due to school locations, the scores of students in schools in central urban areas in all other four dimensions are significantly higher than those of students in schools in suburbs.

The similarities affirm that the current PE teachers in Southwest China attach great importance to the classroom atmosphere, which is the key to ensure enhancement of the teaching quality; as noted by Gillet, the ability to enable students to enjoy PE classes is very important for students to retain the habit of taking exercises in all future life; while for letting students enjoy taking PE classes, the curriculum contents should be varied to meet the students' needs and interests. Therefore, for how to let students learn to cooperate with others in PE classes and meet the students' sports needs and interests, PE teachers may undertake from the curriculum, classroom atmosphere, the image of teacher, obtaining peer acceptance, experiencing achievements and fun in PE classes and other 
aspects. The differences indicate that "No. 7 State Document" has been widely implemented in recent years; the State and local people's governments have increased investment in high school PE expenditure; PE teaching hardware and software in nationwide primary and secondary schools are upgraded comprehensively, so that the teaching environment, facilities and quality have greatly improved, thereby contributing to the promotion of the learning satisfaction of high school students.

The PE learning altitude of high school students in Southwest China can be divided into three dimensions. Seeing from the contribution rates of extracted factors, the three dimensions are "sports awareness" (35.34\%), "sports emotion" (20.54\%) and "sports behavior" (16.55\%) in order. Data analysis showed that the scores of the three dimensions are $3.12 \pm 1.31,3.34 \pm 1.47$ and $3.29 \pm 1.37$ respectively, all above the average. In addition, the scores of "sports emotion" and "sports behavior" have no geographical differences; for "sports awareness", however, the scores of students in central urban areas are significantly higher than that of students in suburbs. On the other hand, we found that for the PE learning attitude of high school students in Southwest China, "sports emotion" scored the highest, indicating students can get obvious benefits from PE classes, such as release of physical and mental pressure, learning to cooperate with classmates and enhancing the physical and mental pleasure. These results agree with findings of Luke and Sinclair, who said the teacher' teaching behavior will affect students' attitude towards PE classes, such as the ability to give students the opportunity to choose and encourage students with poor physical abilities to challenge the self. All these will affect students' attitude toward PE classes.

The correlation results of PE learning satisfaction of students towards their learning attitude showed: the standardized path coefficient was $0.58^{* *}$, reaching a very significant level $(\mathrm{P}<0.01)$, indicating the learning satisfaction of students in PE classes can positively affect their learning attitude. This means "teaching ability", "grounds and equipment", "teaching safety", "sports policy" and "classroom atmosphere" have positive effects on students' "sports awareness", "sports emotion" and "sports behavior". Correlation coefficients show: the five dimensions of learning satisfaction of high school students and the three dimensions of learning attitude are highly correlated mutually. For example, the correlation coefficient of "teaching ability", "grounds and equipment," "teaching safety", "sports policy" and "classroom atmosphere" to "sports awareness" is $0.512 * *, 0.325 * *, 0.387 * *$, $0.419^{* *}$ and $0.614 * *$ respectively, with $0.714^{* *}, 0.413^{* *}, 0.329^{* *}, 0.312^{* *}$ and $0.502 * *$ to "sports emotion" respectively, and $0.534 * *, 0.614 * *, 0.513 * *, 0.417 * *$ and $0.419 * *$ to "sports behavior" respectively. On the other hand, we found that "teaching ability" is highly correlated with "teaching safety" $\left(\mathrm{r}=0.505^{* *}\right)$ and "classroom atmosphere" $\left(\mathrm{r}=0.617^{* *}\right)$, "grounds and equipment" with "teaching safety" $\left(\mathrm{r}=0.413^{* *}\right)$ and "sports policy" $\left(\mathrm{r}=0.345^{* *}\right)$, and "teaching safety" with "classroom atmosphere" ( $\left.\mathrm{r}=0.414^{* *}\right)$.

\section{Conclusion}

Seeing from the contribution rates of issues in the overall PE learning satisfaction of high school students in Southwest China, the "teaching ability" scored the highest, followed by "grounds and equipment", then "teaching safety", and "classroom atmosphere" the lowest. Seeing from scores of five dimensions, the level is basically above the average, with specific performance of "classroom atmosphere" scoring the highest, while the "sports policy" of school scoring the lowest; except the "sports policy" of which the score has no discrepancy caused due to school locations, the scores of students in schools in central urban areas in all other four dimensions are significantly higher than those of students in schools in suburbs.

Seeing from the contribution rates of issues in the overall PE learning altitude of high school students in Southwest China, the "sports awareness" has the greatest contribution, followed by "sports emotion", and "sports behavior" the last one; the scores of "sports emotion" and "sports behavior" have no geographical differences; for the "sports awareness", however, the scores of students in central urban areas are significantly higher than that of students in suburbs.

For high school students in Southwest China, their learning satisfaction in PE classes has a significant positive impact on their learning attitude, with a standardized coefficient of regression and 
path of $0.58^{* *}$; the dimensions of satisfaction and that of learning altitude have significant positive correlation, which means the impacts of "teaching ability", "grounds and equipment", "teaching safety", "sports policy" and "classroom atmosphere" on students' "sports awareness", "sports emotion" and "sports behavior" are positive.

\section{References}

[1]. Gillet,N., Vallerand,R. J., \& Lafreniere, M. K..Intrinsic and extrinsic school motivation as a function of age: The mediating role of autonomy support [J]. Social Psychology of Education: An International Journal, 2012, 15 (33):77-95.

[2]. Hutzler, Y., \& Korsensky, O. Motivational correlates of persons with intellectual disability: A systematic literature review [J]. Journal of Intellectual Disability Research, 2010, 54(13): 767 $-786$.

[3]. Cronin,J.J.,\& Taylor,S.A.Measuring service quality: A reexamination and extension [J].Journal of Marketing, 1992,56(3):55-68.

[4]. Boulding, W.,Kalra, A.,Staelin,R.,\& Zeithaml, V. A. A dynamic process model of service quality: From expectations to behavioral intentions [J].Journal of Marketing Research, 1993, 30(2):7-27.

[5]. Varsamis,P., \& Agaliotis,I. Profiles of self-concept, goal orientation, and self-regulation in students with physical, intellectual, and multiple disabilities: Implications for instructional support[J].Research in Developmental Disabilities, 2011,32(14): 1548-1555.

[6]. Hu, L.T.,\& Bentler, P.M. Cutoff criteria for fit indexes in covariance structure analysis' conventional criteria versus new alternatives[J].Structural Equation Modeling,1999,6(1):45-55. 